\title{
Untersuchungen zu Mensch-Maschine-Schnittstellen eines Pilotenassistenzsystems zur Begegnung potenti- eller Gefahrensituationen der Allgemeinen Luftfahrt
}

\author{
Cheryl Nicoletta Schwahn, Sven Angermann* und Wolfgang Rüther-Kindel
}

\section{Zusammenfassung}

Privatpiloten, die sich in der Allgemeinen Luftfahrt bewegen, sind häufig anderen Gefahren ausgesetzt, als Piloten großer Verkehrsflugzeuge. Gefahrensituationen bringen Piloten schnell an Wissenslücken und Kapazitätsgrenzen. Für Privatpiloten stellt dabei der Kontrollverlust im Flug die häufigste Unfallursache dar.

Das Forschungsvorhaben Virtual Instructor for General Aviation (VIGA) des Fachgebiets Luftfahrttechnik leistet einen wesentlichen Beitrag zur Vermeidung dieser Unfälle. Ziel des Vorhabens ist es, ein Assistenzsystem zu entwickeln, das Privatpiloten frühzeitig mit Handlungsempfehlungen unterstützt, sobald diese in eine unkontrollierte Fluglage gelangen.

Dieser wissenschaftliche Beitrag stellt die Grundlagen des Forschungsvorhabens VIGA dar und beschreibt eine Konzepterstellung zur Mensch-Maschine-Schnittstelle von Assistenzsystemen der zivilen Luftfahrt unter Betrachtung der psychologischen Belastung in Notsituationen. Belastungssituationen beeinträchtigen sowohl die Wahrnehmung der Piloten, beispielsweise durch selektive Taubheit oder optische Täuschungen als auch die variierende Reaktionszeit des Piloten auf verschiedene Reize. Ziel ist es, eine geeignete Mensch-Maschine-Schnittstelle zu entwickeln, die jederzeit die Aufmerksamkeit des Piloten erlangt und Handlungsempfehlungen zielgerecht an diesen ausgibt. Erkenntnisse aus diesem Beitrag finden direkte Anwendung im Vorhaben VIGA.

\section{Abstract}

Private pilots in the field of private transportation have a higher risk to experience an incident than pilots of air carriers. Incidents become hazardous as soon as pilots reach the boundaries of their knowledge and capacities. For private pilots Loss of Control Inflight is the most common cause for an incident.

The research project Virtual Instructor for General Aviation (VIGA) is countering with this problem by creating an assistance system which transmits recommended actions in hazardous situations before a Loss of Control Inflight occurs. This paper summarizes the basic concept of the research project and comprises the concept of human-machine interfaces used in assistance systems in the field of civil aviation. During situations with a high workload the perception is changing. For example, Pilots experience selective auditory attention, visual illusions and a changing response time referring to the attraction. Therefore, it is the aim to create an appropriate human-machine interface which targets the attention and transmits recommended actions to the private pilot. The awareness is used as the foundation of the project VIGA.

\section{Einführung}

Das Forschungsvorhaben Virtual Instructor for General Aviation (VIGA) des Fachgebiets Luftfahrttechnik fokussiert Leichtflugzeuge der Allgemeinen Luftfahrt mit einer maximalen Abflugmasse von 2 t. Dieses Segment wickelt ca. 70 \% der Flugbewegungen in Deutschland ab (Luftfahrt Bundesamt 2018) und spiegelt sich entsprechend in den Unfallstatistiken wider (National Transport Safety Board 2016). Aus diesen stellt sich der Kontrollverlust als häufigste Unfallursache der Allgemeinen Luftfahrt heraus (Federal Aviation Administration 2018). Piloten verlieren dabei die Kontrolle ihrer Luftfahrzeuge und gelangen in eine unkontrollierte Fluglage. Eine stabile Fluglage ist ohne Hilfestellung selten zurückzuerlangen. Menschliches Versagen stellt dabei eine wesentliche Fehlerquelle dar, für das gerade Privatpiloten besonders anfällig sind. 70 \% der Flugunfälle wären durch den Menschen vermeidbar gewesen (National Civil Aviation Review Commission 1998). Assistenzsysteme helfen dabei, diese Fehlerquelle zu reduzieren, indem sie auf Gefahren hinweisen oder konkrete Handlungsempfehlungen an den Piloten bzw. Autopiloten ausgeben. VIGA greift dabei 
jedoch nicht aktiv in die Bedienung des Luftfahrzeuges ein, wie es bei Verkehrsflugzeugen heutzutage üblich ist. Es nutzt in der Luftfahrt bereits erfasste und vielseitig verwendete Parameter für permanente Simulationen während des Fluges. Bei anbahnenden Risiken verwandelt es diese mittels Algorithmen in geeignete Warnsignale und Handlungsempfehlungen.

\section{Risiken von Privatpiloten}

VIGA fokussiert auf Privatpiloten, da diese den menschlichen Risikofaktoren der Luftfahrt stärker ausgesetzt sind als Berufs- oder Verkehrspiloten. Folgende Faktoren begründen diesen Sachverhalt.

Zunächst ist das Ausbildungsniveau erheblich niedriger. Die Ausbildung des Piloten schafft die Grundlage seines Wissens und seiner Erfahrung, auf die er in der Flugdurchführung zurückgreift. Sie ermöglicht inm, mit komplexen Aufgabenstellungen routiniert umzugehen, Entscheidungen effizient zu treffen und schafft mehr Kapazitäten für Situationsbewusstsein. Ein Privatpilot schließt, je nach Ausbildungsform, seine Ausbildung nach 30-45 Flugstunden ab. Berufspiloten benötigen eine Flugerfahrung von 150-200 Flugstunden. Sie werden für komplexere Flugzeugtypen ausgebildet, die technisch besser ausgestattet sind und diverse Assistenzsysteme zur Verfügung haben. Verkehrspiloten müssen eine Erfahrung von mindestens 1.500 Flugstunden nachweisen, bevor sie ein Flugzeug eigenverantwortlich führen dürfen. Bis dahin haben sie zahlreiche Trainings- und Checkflüge absolviert. (Schwahn 2017)

Luftfahrzeuge der Allgemeinen Luftfahrt sind durchschnittlich älter als zehn Jahre und enthalten oft auch einen entsprechend alten Stand der Technik (Erb 2000). Umrüstungen sind kostenintensiv und mit hohen Ausfallzeiten verbunden. Nachteile, die der Flugzeughalter selbst tragen muss.

Ein weiterer Faktor besteht in den Befähigungsüberprüfungen von Piloten. Während Berufspiloten alle sechs Monate einer Befähigungsüberprüfung sowie jährlichen Linechecks unterzogen werden und im Berufsalltag ihre Kenntnisse aufrechterhalten, werden Privatpiloten nur im Abstand von 24 Monaten geprüft (Schwahn 2017). Zusätzlich sind Privatpiloten selbst dafür verantwortlich, an Sicherheitsschulungen in eigenem Ermessen teilzunehmen. Des Weiteren entfällt häufig das geschulte Auge eines Zweimanncockpits, das Teilaufgaben abnimmt oder bestätigt.

Der kommerzielle Luftverkehr weist zudem mehr Personenkilometer, Umsätze und Beschäftigte auf und ist somit attraktiver für die Forschung und Verkehrspolitik. Fluggesellschaften unterliegen stets einem hohen öffentlichen Druck, sobald sich ein Zwischenfall ereignet. Investitionen in Sicherheitsmaßnahmen und Techniken konzentrieren sich daher vorrangig auf die kommerzielle Luftfahrt, zum Nachteil der Privatpiloten.

VIGA hat das Ziel, diesen Risiken durch Hilfestellungen entgegenzuwirken.

\section{Ziel der Arbeit}

Die Zulassungsbehörden legen keinen Standard für die Auslegung von Assistenzsystemen fest, solange sie nicht in die Flugführung bzw. Steuerung eingreifen. Studien und Praxisberichte belegen jedoch, dass Piloten in Situationen mit erhöhter Arbeitsbelastung, unter Zeitdruck, hoher Verantwortung, Lärm- und Mehrfachbelastung diese Systeme nur selektiv wahrnehmen. Die Anforderungen an Pilotenassistenzsysteme bestehen darin, Piloten in Situationen, in denen es ihnen an Erfahrung und Wissen mangelt, zu unterstützen. Das ist nicht gewährleistet, wenn das System den Piloten mit seinen Warnsignalen und Handlungsempfehlungen nicht erreicht. Ziel der Arbeit war es, auf Grundlage von neuroergonomischen Studien eine geeignete Mensch-Maschine-Schnittstelle für das System VIGA zu konzipieren. Es ist erforderlich, diese so auszulegen, dass das System den Piloten auch dann mit unterstützenden Hinweisen erreicht, wenn seine Aufnahmekapazitäten in Notsituationen begrenzt sind. Das Konzept fokussiert auf die Wahrnehmungskanäle, die Piloten während der generellen Flugführung nutzen. Diese umfassen die akustische, visuelle und vestibuläre Wahrnehmung.

\section{Methode und Vorgehensweise}

Die Auslegung von Assistenzsystemen erfolgt nach technischen und psychologischen Rahmenbedingungen sowie Erkenntnissen aus Unfalluntersuchungen. Die theoretischen Grundlagen des Forschungsvorhabens werden durch das Charakterisieren des Anwenderspektrums geschaffen. Dazu gehört es, Unfallraten zu erfassen und daraus die Risiken sowie den Umgang damit zu bestimmen. Risiken und etablierte Maßnahmen werden den Unfalluntersuchungsberichten sowie Luftfahrzeughandbüchern und Lehrbüchern entnommen. Des Weiteren gilt es folgende Fragestellungen zu klären:

\section{Wie können Handlungsempfeh-}

lungen dem Piloten vermittelt werden?

2. Wie sieht die Gestaltung des fertigen Produkts aus?

Dabei werden verschiedene Vorgehensweisen erwogen. Hersteller von Pilotenassistenzsystemen nutzen eigene Labore und Versuchsreihen, um potentielle Mensch-Maschine-Schnittstellen zu testen. Die Untersuchung von Mensch-Maschine-Schnittstellen in bestehenden Assistenzsystemen zeigt, dass für die Aufmerksamkeitsgewinnung vorzugsweise akustische Signale verwendet werden. Sie sind im gesamten Arbeitsumfeld zu empfangen und benötigen gegenüber der visuellen Wahrnehmung keine Konzentration auf ein definiertes Blickfeld. Die Informationen selber werden dann visuell übertragen, um eine selektive Taubheit zu umgehen. Wesentliche Erkenntnisse dazu erarbeitet die Arbeitsgruppe "Menschliche Faktoren und Neuroergonomie" des Instituts für Luft- und Raumfahrt in Toulouse mittels verschiedener Studien (Dehais \& Causse 2017).

\section{Neuroergonomie}

Neuroergonomie erforscht die psychologischen Grundlagen, nach denen Kenntnisse des Arbeitsumfelds mit Aspekten der Neurowissenschaft verbunden werden. Die neuroergonomischen Studien bilden daher auch die Grundlage für die Auslegung der MenschMaschine-Schnittstelle von VIGA und 
die Gestaltung des fertigen Produkts.

Der Mensch steht im Mittelpunkt einer langen Fehlerkette. Die Fehlerkette wird u.a. geprägt durch Verwechslungen, Bedienungsfehler und Missverständnisse. Aufgabe der Hersteller ist es, bereits in der Entwicklung bekannte Fehler zu berücksichtigen und zu kompensieren. Systeme sind an die Leistungsfähigkeit des Menschen aber auch seine Grenzen anzupassen.

Sicherheitsrelevante Erkenntnisse lassen sich in der Luftfahrt zwar meist aus Unfalluntersuchungen gewinnen, der psychologische Hintergrund ist dabei jedoch nur schwer nachvollziehbar. In der Neuroergonomie werden Notsituationen in Studien simuliert. Neue Erkenntnisse werden aus Beobachtungen und Elektroenzephalographie gewonnen. Die Elektroenzephalographie misst Hirnaktivitäten an der Schädeloberfläche mittels rhythmischer Spannungsschwankungen der Nervenzellen. Sie veranschaulicht die Antwort des Gehirns auf Einzelereignisse. Somit ist es möglich, die Aufmerksamkeit auf einen Reiz zu bestimmen und die Wahrnehmung unter erhöhter Arbeitsbelastung zu untersuchen.

\section{Wahrnehmung unter erhöhter Arbeitsbelastung}

Eine Untersuchung der Wahrnehmung des Piloten unter erhöhter Arbeitsbelastung erfordert zunächst die Bestimmung der Wahrnehmung bei der normalen Flugdurchführung. Bei dieser ist die Wahrnehmung des Piloten konzeptgesteuert und routiniert. Dies erfordert eine ganzheitliche Schulung. Dabei dienen Handlungsschemen als Hilfestellung, um komplexe Aufgabenstellungen zu erfüllen. Unerfahrene Piloten können bereits bei der normalen Flugdurchführung an ihre Kapazitätsgrenzen gelangen. Flugdurchführungen mit Störfällen können auch bei geschulten Piloten Stress auslösen. Sinne und Reize werden verändert wahrgenommen. Das Handeln erfolgt dann abseits von geschulten Abläufen. Die Auswirkung von Störfällen auf die Psychologie des Menschen variiert und ist nicht umfassend voraussehbar. Die Arbeitsgruppe "Menschliche Faktoren und Neuroergonomie“ des Instituts für
Luft- und Raumfahrt in Toulouse führte verschiedene Studien durch, die die Wahrnehmung von Menschen unter verschiedenen Bedingungen untersuchen (Dehais \& Causse 2017). Diese werden nachfolgend erläutert.

\section{Verlust der auditiven Wahrnehmung}

T. P. Newman schilderte 1993 in "The philosophy of aircraft warning systems", dass Flugschüler in Situationen mit hoher Arbeitsbelastung zunächst ihre auditive Wahrnehmung verlieren. Sie reagieren weder auf Anweisungen des Fluglehrers noch auf Kontrollfreigaben durch Fluglotsen (Newman 1993). Die Arbeitsgruppe testete dieses Szenario, indem sie Probanden aufforderte, akustische Signale, die von der visuellen Anzeige abweichen, per Knopfdruck zu detektieren. Dabei wurden $31 \%$ der fehlerhaften Signale nicht wahrgenommen (Dehais \& Causse 2017).

\section{Wahrnehmung korrespondierender} gegenüber abweichenden Signalen

Eine weitere Studie dieses Instituts untersuchte den Zusammenhang der Wahrnehmung von akustischen und visuellen Signalen. Die Aufgabenstellung bestand darin, die Entscheidung zur Landung anhand von visuellen und akustischen Signalen abzuwägen. Bei der ersten Versuchsreihe korrespondierten akustisches und visuelles Signal und versprachen eine problemlose Landung. Die Probanden folgten dem Signal. In einer weiteren Versuchsreihe war gemäß dem visuellen Signal eine Landung durchführbar, während die akustische Warnung das Gegenteil signalisierte. Die Probanden führten die Landung durch und vertrauten damit dem visuellen Signal. Abb. 1 stellt die Ergebnisse der Studie und die dazugehörigen Aufnahmen der Elektroenzephalographie dar. Die Aufnahmen veranschaulichen, dass das akustische Signal intensiver in Begleitung mit einem korrespondierenden visuellen Signal wahrgenommen worden ist. Stimmten die Aussagen beider Signale nicht überein, wurde das akustische Signal kaum wahrgenommen (Dehais \& Causse 2017).

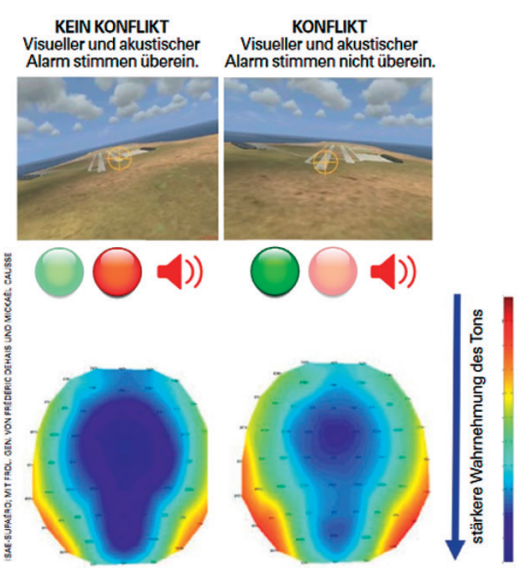

Fig. 1) Aufnahmen der Versuchsreihe zur akustischen Wahrnehmung (Dehais \& Causse 2017).

Akustische Wahrnehmung unter variierendem Schwierigkeitsgrad

In einer weiteren Untersuchung wurde die Wahrnehmung akustischer Signale unter variierendem Schwierigkeitsgrad getestet. Probanden erhielten die Aufgabe, eine Landung durchzuführen. Zeitgleich wurde innen ein wiederkehrender Ton vorgespielt, der unregelmäßig mit einem hohen Ton versetzt wurde. Der hohe Ton musste von den Probanden über einen Knopf bestätigt werden. Von den hohen Tönen wurden 20 \% nicht bestätigt. Wurden die Landebedingungen zusätzlich erschwert, wurden 30 \% der Töne nicht wahrgenommen. Daraus lässt sich schließen, dass die selektive Taubheit mit steigender Belastung zunimmt. (Dehais \& Causse 2017)

Wahrnehmung akustischer Reize unter variierender Belastung

Mit derselben Problematik beschäftigte sich eine weitere Studie des Instituts. Indikator für die Untersuchung war die P300-Welle aus der Elektroenzephalographie. Sie gibt Auskunft über den Zeitabschnitt und die Intensität der Hirnaktivitäten, die ein Reiz hervorruft. Generell liegt die Antwortzeit zwischen 300-700 ms bei gesunden Menschen. Abb. 2 veranschaulicht die Ergebnisse der Untersuchung. Die blaue Kurve veranschaulicht die Reizwahrnehmung in ruhiger Arbeitsatmosphäre. Der Reiz wird intensiv wahrgenommen. Die grüne Kurve zeigt eine deutlich schwächere Intensität der Reizwahrnehmung. Bei diesem Versuch wurden die 
Probanden visuell abgelenkt. Während einer weiteren Versuchsdurchführung wurde zudem eine Notsituation simuliert. Die rote Kurve verdeutlicht dabei, dass der akustische Reiz kaum wahrgenommen wurde. (Giraudet et al. 2015). eine Stresssituation zu erzeugen. In beiden Versuchsgruppen verringerte sich die Qualität der erbrachten Leistung mit steigendem Schwierigkeitsgrad. Die Elektroenzephalographie gab Aufschluss über die Unterschiede

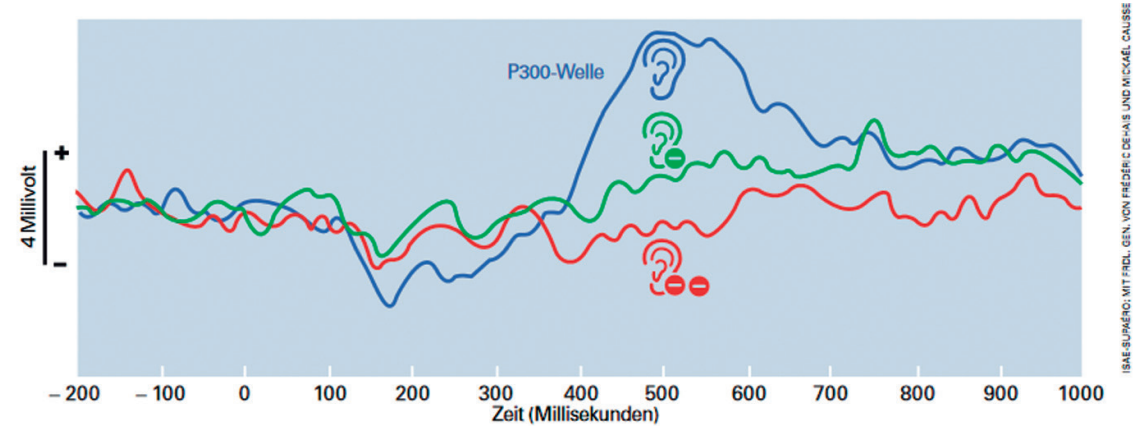

Abb. 2) Wahrnehmung akustischer Signale unter variierender Arbeitsbelastung (Dehais \& Causse 2017).

Visuelle Wahrnehmung unter variierender Arbeitsbelastung

Ein weiterer Versuch testete die visuelle Wahrnehmung der Probanden unter erhöhter Arbeitsbelastung. Die Aufgabe bestand darin, anhand einer Drohnenaufnahme eine Zielscheibe zu erkennen. Die Versuchsgruppe war dabei so fixiert auf ihre Aufgabe, dass ein eingeblendeter leerer Akkustand nicht bemerkt wurde. Die Studie wurde fortgeführt, indem die Drohnenaufnahme kurzzeitig ausgeblendet wurde, so dass der Bildschirm lediglich den leeren Akkustand signalisierte. Dabei wurden sämtliche Probanden auf das Problem aufmerksam. Der Versuch verdeutlicht, dass die Informationsaufnahmekapazitäten des Menschen beschränkt sind. (Dehais \& Causse 2017)

\section{Bedeutung des präfrontalen Kortex} in der Wahrnehmung

Der präfrontale Kortex im Stirnhirn sorgt dafür, dass der Mensch in der Lage ist, mehrere Reize zu priorisieren und Stress in geringem Maße zu kompensieren. Probanden wurden in einer Versuchsreihe des Instituts dazu aufgefordert, Aufgabenstellungen mit steigerndem Schwierigkeitsgrad zu bewältigen. Die Aufgabe bestand darin, die Durchführung eines Durchstartmanövers abzuwägen. Eine weitere Versuchsgruppe bekam dieselbe Aufgabenstellung, wurde vor dem Versuch jedoch auf die Kosten eines Durchstartmanövers hingewiesen, um dadurch

beider Versuchsgruppen, dargestellt in Abb. 3. Die Versuchsgruppe, die einer erhöhten Stresssituation ausgesetzt wurde, weist in der Elektroenzephalographie einen deutlich stärker angeregten präfrontalen Kortex vor. Die Aufgabenbewältigung unterschied sich in beiden Versuchsgruppen nicht voneinander. Psychologen gehen deshalb davon aus, dass das Gehirn in der Lage ist, Stress in geringem Maß zu kompensieren. (Causse et al. 2013)



Abb. 3) Aktivitäten des präfrontalen Kortex während einer Belastungsstudie (Dehais \& Causse 2017).

\section{Nutzbarkeit von Spiegelneuronen}

Spiegelneuronen stellen eine wesentliche Hilfestellung in der Informationsaufnahme und -verarbeitung des Menschen dar. Kinder nutzen diese bei- spielsweise, um Verhaltensweisen ihrer Umgebung zu adaptieren und $u$. a. das Sprechen zu erlernen. Erwachsene, die Verhaltensweisen ihrer Gesprächspartner nachahmen, erzielen Sympathie bei diesen. Spiegelneuronen sind im Cockpit ebenfalls nutzbar. Probanden bekamen die Aufgabe, Handlungsempfehlungen einer Animation umzusetzen. Die Animationen aktivierten die Nervenzellen der Probanden direkt und die Reaktionszeit verkürzte sich um $60 \%$. (Callan et al. 2012)

\section{Schlussfolgerungen}

Piloten des Anwenderspektrums benötigen ein Tool, dass sie in Notsituationen mit Handlungsempfehlungen unterstützt, da sie unter Stress oftmals abseits ihrer geschulten Arbeitsabläufe handeln. Um Handlungsempfehlungen ausgeben zu können, ist es zunächst erforderlich, die Aufmerksamkeit des Piloten zu erlangen. Akustische Signale sind hierfür grundsätzlich geeignet, da sie im gesamten Arbeitsumfeld wahrgenommen werden können und nicht die Konzentration auf ein definiertes Blickfeld erfordern. Die Studien verdeutlichen jedoch, dass der 
daher, akustische Signale mit visuellen, korrespondierenden Signalen zu koppeln. Visuelle Signale sind dabei so darzustellen, dass sämtliche Informationen eines Panels, die einer niedrigeren Priorität entsprechen, ausgeblendet werden, um beim Piloten Aufnahmekapazitäten für neue Informationen frei zu machen. Handlungsempfehlungen lassen sich visuell besser darstellen und vom Piloten vollständig erfassen. Komplexe Darstellungen sind somit umfassend aufführbar. Um Missverständnisse zu vermeiden, eignen sich zusätzliche Empfehlungen in Textform. Diese können mit Animationen gekoppelt werden, um die Spiegelneuronen des Piloten zu nutzen und die Reaktionszeit zu verkürzen.

\section{Ausblick auf das künftige Produkt}

Das fertige Produkt soll in der Lage sein, auf Unregelmäßigkeiten akustisch und visuell hinzuweisen und Handlungsempfehlungen durch visuelle Reize in Textform und per Animation auszugeben.

Piloten nutzen im Allgemeinen Headsets zur Kommunikation aber auch zum Lärmschutz, da die Umgebungsgeräusche in einem Leichtflugzeug hoch sind. Damit akustische Signale den Piloten erreichen, sollten sie direkt über das Headset ausgegeben werden. Besonders geeignet erscheinen dazu dynamische Headsets, die Umgebungsgeräusche aktiv unterdrücken und damit die Aufmerksamkeit weiter fokussieren. Ein Bespiel für ein solches Headset ist das A20 Aviation Headset des Herstellers Bose, dargestellt in Abb. 4. Durch Anschlussmöglichkeiten über AUX und Bluetooth bietet es die Möglichkeit, verschiedene Systeme, die in das Headset eingespeist werden, zu priorisieren.
Die visuelle Ausgabe muss die Möglichkeit bieten, Texte und Animationen darzustellen. Bevor eine Handlungsempfehlung ausgegeben wird, sollte sichergestellt sein, dass die Aufmerksamkeit des Piloten erlangt wurde. Dementsprechend wird ein Ausgabeund Bedienelement benötigt. Diese Problemstellung lässt sich durch ein Tablet mit Touch-Funktion lösen. Als solches bietet sich z.B. das RugGear-Tablett an. Es ist robust und unempfindlich gegenüber Störungen. Anschlussmöglichkeiten bestehen über USB.

Die Mensch-Maschine-Schnittstelle erreicht den Piloten somit entsprechend dessen Wahrnehmung in Notsituationen gemäß neuroergonomischer Untersuchungen. Die Wahrnehmung variiert in Notsituationen jedoch zwischen den Piloten, somit lässt sich eine hundertprozentige Sicherheit nicht gewährleisten.

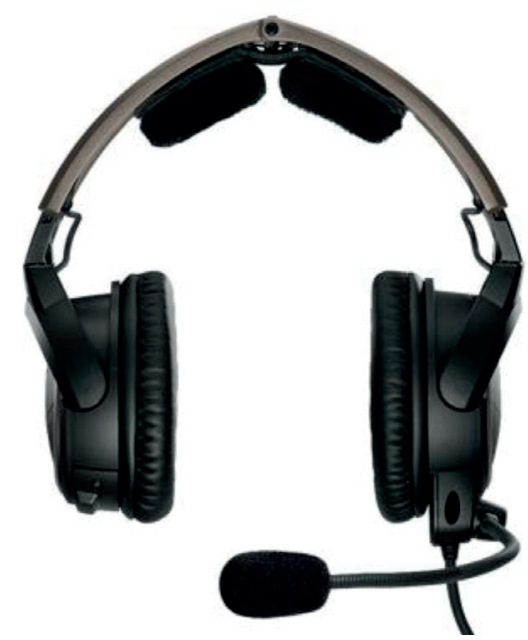

Abb. 4) Headset A20 Aviation (Bose, n. d.).

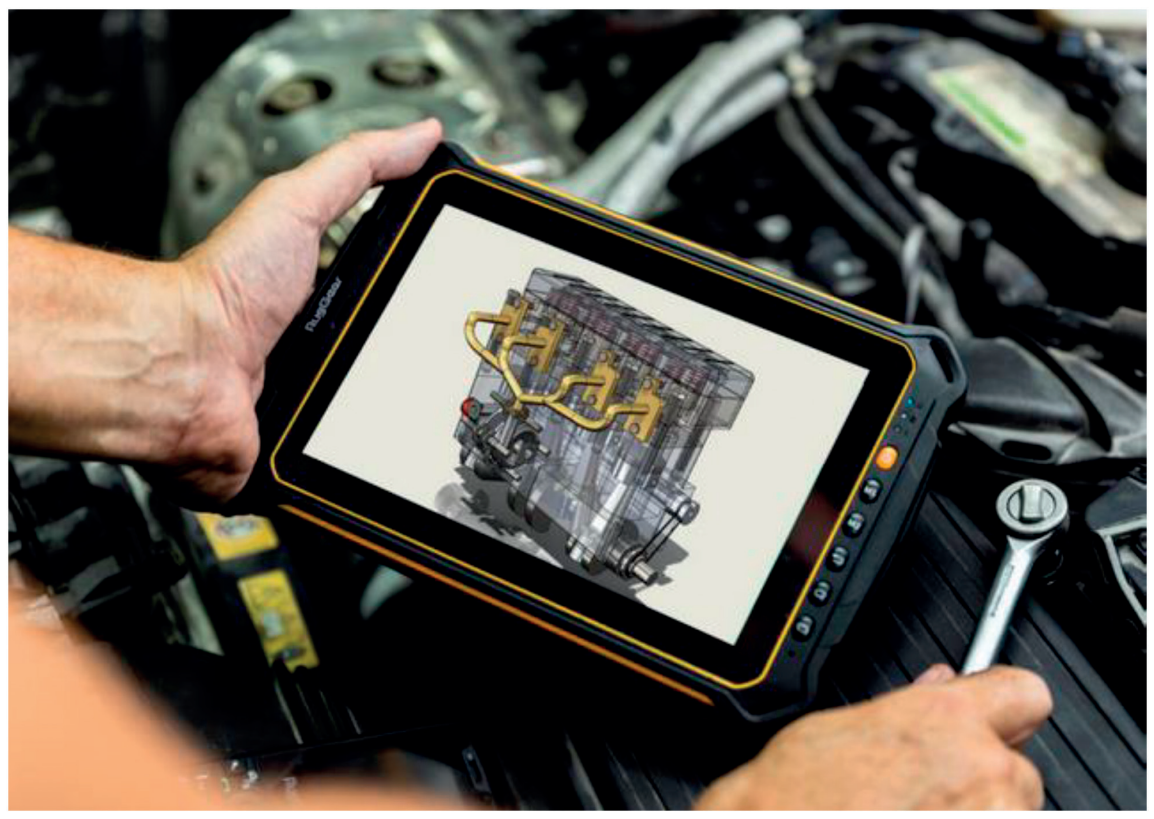

Abb. 5) RugGear Tablet mit Touch-Funktion (RugGear, n. d.). 


\section{LITERATUR}

Aircraft Owners and Pilots Association AOPA (2016) Allgemeine Luftfahrt: Was ist das?

Callan DE, Gamez M, Cassel DB, Terzibas C, Callan A, Kawato M, Sato M-a (2012) Dynamic visuomotor transformation involved with remote flying of a plane utilizes the 'Mirror Neuron' system. PLoS one 7(4):e33873.

doi: 10.1371 /journal.pone. 0033873

Causse M, Péran P, Dehais F, Caravasso CF, Zeffiro T, Sabatini U, Pastor J (2013) Affective decision making under uncertainty during a plausible aviation task: an

fMRI study. Neuroimage 71:19-29. doi: 10.1016/j.neuroimage.2012.12.060

Dehais F, Causse M (2017) Entscheiden im Flugmodus. Spektrum der Wissenschaft 2017(11)

Dehais F, Roy R, Durantin G, Thibault G, Callan D (2017) EEG-engagement index and auditory alarm misperception: an inattentional deafness study in actual flight condition. In: Advances in Neuroergonomics and Cognitive Engineering: Proceedings of the AHFE 2017 Internationa Conference

Erb M (2000) Die allgemeine Luftfahrt in Deutschland: eine Analyse im Spannungsfeld von Ökonomie und Ökologie. Dissertation, TU Darmstadt

Federal Aviation Administration (2009) Risk Management Handbook. FAA-H-8083-2

Federal Aviation Administration (2018) Fact Sheet - General Aviation Safety.

Gateau T, Durantin G, Lancelot F, Scannella S, Dehais $F(2015)$ Real-time state estimation in a flight simulator using fNIRS. PLoS one 10(3):e0121279. doi: 10.1371/ journal.pone.0121279

Giraudet L, St-Louis M-E, Scannella S, Causse M (2015) P300 event-related potential as an indicator of inattentional deafness? PLoS one 10(2):e0118556. doi: 10.1371/ journal.pone. 0118556

Gleim IN (2012) Pilot handbook. A comprehensive text/ reference for all pilots, 6 th ed. Gleim Publications, Gainesville, Fla.

Luftfahrt Bundesamt (2018) Bestand an Luftfahrzeugen in der Bundesrepublik Deutschland.

Moir I, Seabridge AG (2008) Aircraft systems. Mechanical, electrical, and avionics subsystems integration. Wiley,

Chichester, West Sussex, England, Hoboken, N.J. ISBN: 9780470059968 . doi: $10.1002 / 978047077093$

National Civil Aviation Review Commission (1998) Avoiding aviation gridlock \& reducing the accident rate: a consensus for change. Washington, D.C.

National Transportation Safety Board (2016) 2014 NTSB US Civil Aviation Accident Statistics. Aviation: Data \& Stats

Newman TP (1993) The Philosophy of Aircraft

Warning Systems. Proceedings of the Institution of Mechanical Engineers, Part G: Journal of Aerospace Engineering 207(2):75-82. doi: 10.1243/PIME

PROC_1993_207_251_02

Scheiderer J, Ebermann H-J (eds) (2011) Human Factors im Cockpit. Praxis sicheren Handelns für Piloten. Springer, Berlin. ISBN: 9783642151675

Schwahn CN (2018) Konzept zur Umsetzung von Handlungsempfehlungen in potentiellen Gefahrensituationen der Allgemeinen Luftfahrt. Bachelorarbeit, Technische Hochschule Wildau

Schwahn K-J (2017) Beruf Pilot. Voraussetzungen, Ausbildung, Alltag. Motorbuch-Verl., Stuttgart. ISBN: 9783613028951

\section{AUTOREN}

Prof. Dr.-Ing. Wolfgang Rüther-Kindel Dr.-Ing. Sven Angermann

Cheryl Nicoletta Schwahn, B. Eng.

Fachgebiet Luftfahrttechnik

Luftfahrttechnik / Luftfahrtlogistik

Technische Hochschule Wildau

E-Mail für Korrespondenz:

sven.angermann@th-wildau.de



